\title{
Suprachiasmatic Nucleus Interaction with the Arcuate Nucleus; Essential for Organizing Physiological Rhythms
}

\author{
DiDrederik N. Buijs, ${ }^{1}$ Mara Guzmán-Ruiz, ${ }^{1}$ [Duis León-Mercado, ${ }^{1}$ (D) Mari Carmen Basualdo, ${ }^{1}$ \\ (1) Carolina Escobar, ${ }^{2}$ () Andries Kalsbeek, ${ }^{3}$ and ${ }^{-}$Ruud M. Buijs ${ }^{1}$
}

DOI:http://dx.doi.org/10.1523/ENEURO.0028-17.2017

${ }^{1}$ Instituto de Investigaciones Biomedicas, UNAM, Ciudad Universitaria, 04510 Mexico DF, Mexico, ${ }^{2}$ Departamento de Anatomia, Facultad de Medicina, UNAM, Ciudad Universitaria, 04510 Mexico DF, Mexico, ${ }^{3}$ Netherlands Institute for Neuroscience, an Institute of the Royal Netherlands Academy of Arts and Sciences, Amsterdam 1105 BA, The Netherlands

\begin{abstract}
The suprachiasmatic nucleus (SCN) is generally considered the master clock, independently driving all circadian rhythms. We recently demonstrated the SCN receives metabolic and cardiovascular feedback adeptly altering its neuronal activity. In the present study, we show that microcuts effectively removing SCN-arcuate nucleus (ARC) interconnectivity in Wistar rats result in a loss of rhythmicity in locomotor activity, corticosterone levels, and body temperature in constant dark (DD) conditions. Elimination of these reciprocal connections did not affect SCN clock gene rhythmicity but did cause the ARC to desynchronize. Moreover, unilateral SCN lesions with contralateral retrochiasmatic microcuts resulted in identical arrhythmicity, proving that for the expression of physiological rhythms this reciprocal SCN-ARC interaction is essential. The unaltered SCN c-Fos expression following glucose administration in disconnected animals as compared to a significant decrease in controls demonstrates the importance of the ARC as metabolic modulator of SCN neuronal activity. Together, these results indicate that the SCN is more than an autonomous clock, and forms an essential component of a larger network controlling homeostasis. The present novel findings illustrate how an imbalance between SCN and ARC communication through circadian disruption could be involved in the etiology of metabolic disorders.
\end{abstract}

Key words: circadian rhythm; clock genes; melatonin; network; oscillator coupling; temperature

\section{Significance Statement}

The suprachiasmatic nucleus (SCN) is generally considered the master clock, independently coordinating all circadian rhythms. The present study challenges that view and shows that for a proper functioning circadian system, SCN interaction with the arcuate nucleus (ARC) is essential. We observed that interruption of SCN-ARC communication does not affect SCN rhythmicity but causes the ARC to desynchronize. This desynchrony between SCN and ARC results in animals losing activity, temperature, and corticosterone rhythmicity. As the ARC is an essential metabolic integration center, this might explain how chronic circadian or metabolic disruptions through ill-timed eating habits or shift work could cause desynchrony among hypothalamic oscillators and result in disease.

\section{Introduction}

The suprachiasmatic nucleus (SCN) receives light input through direct synaptic connections from the retina; these

Received January 25, 2017; accepted March 11, 2017; First published March 17, 2017.

The authors declare no competing financial interests. photic cues attune the SCN rhythm with the environment. The SCN neuronal network consists of $\sim 20,000$ tightly coupled neurons (Webb et al., 2009), with most neurons 
expressing their own endogenous rhythm through phasic clock gene expression (Reppert and Weaver, 2002). It is proposed that through multi phasic relationships between neuronal oscillators within the SCN, different physiologic rhythms can be timed and entrained to follow circadian patterns (Quintero et al., 2003; Kriegsfeld et al., 2004). In view of existing interactions between different hypothalamic nuclei and the SCN, one could presume the SCN as element of the hypothalamus in a similar fashion, i.e., the SCN not only leading but taking part in a hypothalamic network of coupled oscillators generating circadian rhythmicity. Indeed, the SCN receives a myriad of nonphotic input, arousal (Antle and Mistlberger, 2000), feeding behavior (Abe et al., 1989; Mendoza, 2007), locomotor activity (Edgar et al., 1991; Marchant and Mistlberger, 1995), immune function (O'Callaghan et al., 2012; GuerreroVargas et al., 2014), blood pressure (Peters et al., 1994; Buijs et al., 2014), and melatonin (Armstrong, 1989; Pitrosky et al., 1999), which are all able to adjust and synchronize the SCN. The SCN is capable of receiving this feedback through its large array of reciprocal neuronal connections with, e.g., the arcuate nucleus (ARC; Saeb-Parsy et al., 2000; Yi et al., 2006), intergeniculate leaflet (IGL; Janik and Mrosovsky, 1994; Saderi et al., 2012), nucleus tractus solitarius (Buijs et al., 2014), dorsal raphe (Shioiri et al., 1991), and dorsomedial hypothalamus (DMH; Acosta-Galvan et al., 2011), allowing these nuclei to convey nonphotic feedback to the SCN and thus adjusting circadian rhythmicity. Therefore, it is enticing to propose that the SCN is part of or central in a larger, complex neuronal network regulating circadian physiology and behavior.

We thus hypothesized that generating and synchronizing physiologic circadian rhythms depends on the integration of photic and nonphotic feedback to the SCN through existing strong neuronal interconnectivity with other hypothalamic nuclei. In view of recent observations that lesions targeted at specific neuronal populations in the ARC resulted in deteriorated temperature, feeding, and sleep rhythms (Li et al., 2012; Wiater et al., 2013), and the observations that the SCN induces a rhythm in the ARC (Guzmán-Ruiz et al., 2014; Herrera-Moro et al., 2016), and the ARC influences the activity of the SCN (Yi et al., 2006), we hypothesized that ARC-SCN reciprocity is an essential feedback pathway for the SCN, adjusting its output and facilitating changes in its neural activity in response to physiologic and behavioral activity. To test

This work was supported by the CONACyT Grant 220598 and the DGAPAUNAM Grant IG-200417.

Acknowledgments: We thank Prof. Dr. D. Swaab for his thoughtful comments and review of this manuscript and Wilma Verwey for her work in the revision of English language.

Correspondence should be addressed to Ruud M. Buijs, Hypothalamic Integration Mechanisms, Instituto de Investigacíones Biomedicas, Universidad Nacional Autonoma de Mexico, 04510 Mexico DF, Mexico. E-mail: ruudbuijs@ gmail.com.

DOl:http://dx.doi.org/10.1523/ENEURO.0028-17.2017

Copyright $\odot 2017$ Buijs et al.

This is an open-access article distributed under the terms of the Creative Commons Attribution 4.0 International license, which permits unrestricted use, distribution and reproduction in any medium provided that the original work is properly attributed. this hypothesis, we made retrochiasmatic knife cuts to isolate the ARC from the SCN, preventing their reciprocal communication; we measured specific physiologic output, along with central and peripheral clock gene expression. Under constant dark (DD) conditions such an SCNARC dissection resulted in animals showing complete arrhythmicity in behavior, temperature, and corticosterone secretion despite a rhythmic SCN and persistent rhythmic melatonin secretion. Unilateral SCN lesions combined with contralateral knife cuts provided identical outcomes. Immunohistochemistry and neuronal tracing confirmed successful disconnection of the SCN-ARC interaction. In addition, we demonstrated the IGL-SCN projections and SCN-subparaventricular zone (SPZ), paraventricular nucleus (PVN) and dorsomedial hypothalamic $(\mathrm{DMH})$ projections to be unaffected by the knife cuts. In conclusion, the present results demonstrate the interaction between SCN and ARC to be crucial for the expression of select circadian rhythms and that the circadian network extends beyond the boundaries of the SCN.

\section{Materials and Methods}

\section{Animals and ethical approval}

Experiments were performed on male Wistar rats ( 250g) housed individually on a 12/12 h light/dark (LD) cycle (lights on, 7 A.M.) in a controlled environment. Rats were given food and water ad libitum unless otherwise stated. Piezoelectric motion sensors underneath the cages monitored locomotor activity and temperature data were acquired using ibuttons (Maxim) surgically inserted into the abdominal cavity.

\section{Surgery}

All animals undergoing surgery were anesthetized with ketamine $(50 \mathrm{mg} / \mathrm{kg}$ ) and xylazine $(2 \mathrm{mg} / \mathrm{kg}$ ) (PisaAgropecuaria S.A. de C.V.). Initial preliminary experiments were performed using bilateral thermic ARC lesions using two Teflon coated electrodes $0.2 \mathrm{~mm}$ in diameter with excoriated tip. Animals were placed in a stereotactic frame (toothbar, $-4 \mathrm{~mm}$; arm, $6^{\circ}$; coordinates: -2.0 from bregma; \pm 1.2 lateral from midline; $8.2-8.6 \mathrm{~mm}$ below brain surface) and an electric current of $0.3 \mathrm{~mA}$ was passed for $60 \mathrm{~s}$, sufficient to eliminate the ARC bilaterally.

Medial, retrochiasmatic knife cuts (RC cut) were made using a small knife derived from two telescoped needles with a $45^{\circ}$ angle, sharpened tip. RC cuts were intended to disrupt fibers of passage, without causing extensive damage to the neuronal populations in the retrochiasmatic area. Animals were placed in a stereotactic frame (toothbar, $-4 \mathrm{~mm}$; knife placed perpendicular to midline, -1.1 $\mathrm{mm}$ from bregma), the knife was lowered ventrally until the sphenoid bone was reached $(\sim 8.2 \mathrm{~mm}$ below brain surface) and turned $90^{\circ}$ caudally, in duplex (Fig. 1A,C). In SHAM animals, the surgical procedure was identical but the knife lowered without gyrating.

To further investigate the phenotype resulting from the disrupted interaction between SCN and ARC, unilateral SCN lesions were made, jointly with a contralateral $90^{\circ}$ knife cut $\left(\mathrm{SCN}_{\mathrm{X}} \mathrm{ARC} \mathrm{C}^{\mathrm{Cut}}\right.$; Fig. $\left.1 B, D\right)$. Animals were subjected to the same experimental protocols as animals 


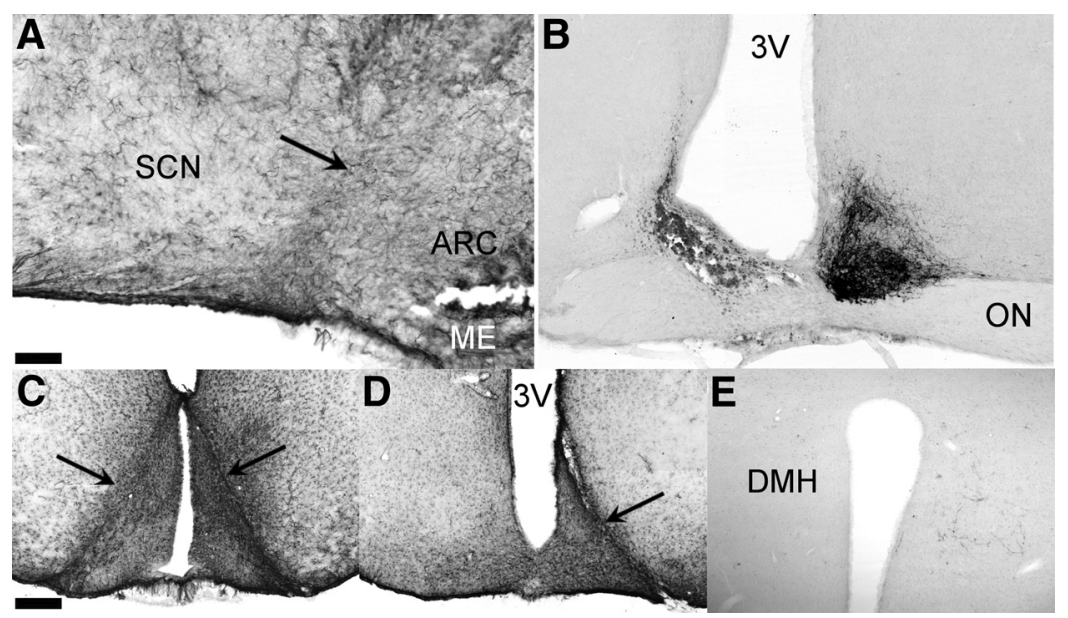

Figure 1. Representative sagittal and coronal sections of the hypothalamus illustrating the $45^{\circ}$ angle knife cut and its effect established through GFAP and VIP staining. A, Sagittal GFAP-stained section just lateral to the third ventricle with minimal glial damage around the site of incision indicated by a black arrow. $\boldsymbol{B}$, Unilateral SCN lesion in combination with a contralateral knife cut with VIP staining showing the contralateral SCN intact. $\boldsymbol{C}$. Shows GFAP staining of the most caudal reach of the knife isolating the ARC from the SCN. $\boldsymbol{D}$, Unilateral RC-cut isolating the ARC contralateral to the SCN lesion shown in $B$. $\boldsymbol{E}$, Unilateral VIP innervation of the $\mathrm{DMH}$ on the side of the RC-cut demonstrating effective unilateral innervation as compared with the loss of innervation on the SCN-lesioned side (left). Scale bar, $100 \mu \mathrm{m}(\boldsymbol{A}), 90 \mu \mathrm{m}(\boldsymbol{B}), 250 \mu \mathrm{m}(\boldsymbol{C}$ and $\boldsymbol{D})$, and $130 \mu \mathrm{m}(\boldsymbol{E})$. ME, median eminence; 3V, third ventricle.

receiving retrochiasmatic knife cuts (RC cut). Finally, we performed a glial fibrillary acidic protein (GFAP) immunostaining to show the extent of glial scarring, thus assessing the magnitude of damage caused by placed knife cuts. This demonstrated only minor glial damage to adjacent areas in RC-cut-operated animals (Fig. $1 A, C, D$ ).

In testing to what degree severing SCN-ARC efferents eliminate neuronal innervation of the SCN, we performed unilateral cholera toxin B (CtB), ARC injections in animals with and without ipsilateral retrochiasmatic knife cut. CtB injections (Invitrogen) were made using the above coordinates for the ARC. With a glass micropipette $(\sim 30-\mu \mathrm{m}$ tip), $0.05 \mu \mathrm{l} 1 \% \mathrm{CtB}$ was pressure injected (10 mbar, $5 \mathrm{~s}$ ) and the micropipette left in place for $5 \mathrm{~min}$ to minimize tracer leakage.

\section{Perioperative protocol}

Before surgery, a 7 d LD/7 d DD baseline was acquired, and unless stated otherwise, all animals were kept under a 12 h [6 A.M. lights on (300 lux), 6 P.M. lights off] LD cycle. After surgery, a similar protocol was adhered to. Experimental (both $\mathrm{RC}$ cut and $\mathrm{SCN}_{\mathrm{X}} \mathrm{ARC} \mathrm{C}^{\mathrm{Cut}}$ animals) and SHAM animals were allowed to recover and kept in L/D for 8-10 d, followed by $7 \mathrm{~d}$ of DD, during which activity and temperature recordings were made. Of all animals operated ( $n=49$; RC-cut, SHAM and SCNxARCx animals) a total of 40 were included for further analysis. Only animals showing arrhythmic locomotor activity in DD through $\chi^{2}$ analysis and in which subsequent anatomic analysis demonstrated the knife cut to be placed medially, in the retrochiasmatic area, were included. In experiments performed during LD conditions, time is indicated as zeitgeber time (ZT), as the animals are entrained to light (zeitgeber). Experiments performed in DD conditions, time is indicated as circadian time (CT), indicating the animals endogenous rhythm of $\sim 24 \mathrm{~h}$ (circa dies) not entrained to external cues.

\section{Blood sample collection}

Blood was drawn $(200 \mu \mathrm{l})$ from the tail at ZT and CT 0 , 6,12 , and 18; centrifuged for $5 \mathrm{~min}$ at $5000 \mathrm{rpm}$, and supernatant was frozen at $20^{\circ} \mathrm{C}$, from which melatonin (IBL International) and corticosterone were assayed using an ${ }^{125}$ I RIA kit (MP Biomedicals). Rats were placed in a towel during blood withdrawal to minimize handling stress and they were habituated daily for $5 \mathrm{~d}$ before blood withdrawal; care was taken to draw blood within $30 \mathrm{~s}$ after taking the animal out of its home cage. Blood was sampled at CT/ZT 0 and 12 on the same day. At least $2 \mathrm{~d}$ later, blood was sampled at 6 and 18 .

\section{Physiologic rhythmicity following SCN-ARC axis disruption}

The different groups of animals were treated as follows. Preliminary pilot experiments were performed using bilateral thermic ARC lesions $(n=3)$. Thereafter, RC-cut $(n=$ $16)$ and SHAM-operated animals $(n=18)$ were exposed to above-mentioned LD and DD conditions, during which locomotor activity and temperature were registered $(n=7$ for both RC-cut and SHAM animals). The remaining animals ( $n=9$ for RC-cut and $n=11$ for SHAM) were used for immunohistochemistry and in situ analysis. Following this initial registration period and to evaluate melatonin and corticosterone levels, two hormones reflecting direct influence of the SCN, blood samples were taken in LD at ZT0, ZT6, ZT12, and ZT18 ( $n=5-7 /$ time point). Blood was drawn from the tail $2 \mathrm{~d}$ apart at two different time points per animal to minimize stress. One week later, blood was drawn from each animal for DD blood serum analysis following $36 \mathrm{~h}$ of DD at CT0, CT6, CT12, and CT18 $(n=$ $3-5 /$ time point). Then animals were killed using an over- 
dose of sodium pentobarbital (Sedal-Vet $65 \mathrm{mg} / \mathrm{ml}$ ) livers were extracted, immediately put on dry ice and kept frozen at $-80^{\circ} \mathrm{C}$ until further analysis, then animals were perfused transcardially with saline, followed by a freshly prepared $4 \%$ paraformaldehyde solution. Following perfusion fixation, brains were removed and preserved for histologic analysis and in situ hybridization (ISH), as stated below.

$\operatorname{SCN}_{x} A C^{\text {Cut }}$ animals $(n=6)$ underwent a similar protocol and likewise blood samples were taken at ZTO, ZT6, ZT12, and ZT18 ( $n=5-6 /$ time point). Due to technical issues we could record a full temperature registry of only four animals. Animals were killed and their brains extracted for immunohistochemistry to verify the extent of the lesion and knife cut.

\section{Testing the ARC-SCN interaction}

To investigate the relevance of the feedback from the ARC to the SCN, we assessed whether metabolic signaling to the ARC would differently affect SCN activity, depending on whether animals received feedback from the ARC or not, allowing us to demonstrate that the ARC directly alters SCN neuronal activity in response to metabolic signals. RC-cut-operated rats were fasted for $48 \mathrm{~h}$ in LD (12 h lights on/off) followed by $5 \mathrm{ml}$ of $3 \%$ oral glucose $(0.05-\mathrm{g}$ glucose $/ \mathrm{kg})$ intake given in a separate water bottle at ZT2 and killed $2 \mathrm{~h}$ later (ZT4). In two other groups, SHAM-operated $(n=4-6)$ and RC-cut animals ( $n=4-6)$, animals were $48 \mathrm{~h}$ fasted or given ad libitum conditions. Before fasting, only animals receiving glucose were entrained to drink $3 \%$ glucose water during the light period at random time points during $5 \mathrm{~d}$. All animals were killed at ZT4. In brain sections of the differently treated animals, the neuronal activity of the SCN and ARC was examined using c-Fos as activity marker.

\section{Immunohistochemistry}

Following killing, brains were removed, post fixed for $24 \mathrm{~h}$, cryoprotected in $30 \%$ sucrose for $48-72 \mathrm{~h}$, frozen, and cut in coronal sections of $35 \mu \mathrm{m}$ at $-20^{\circ} \mathrm{C}$. Free floating sections were processed for c-Fos (rabbit polyclonal, 1:40,000; Calbiochem), $\alpha$-MSH (sheep, 1:10,000; Millipore Bioscience Research Reagents), AgRP (goat, 1:4000; Millipore Bioscience Research Reagents), VIP (rabbit, 1:2000; Buijs et al., 1989), Per1 (rabbit 1:2000, Santa Cruz Biotechnology), and CtB (rabbit, 1:2000; Sigma-Aldrich) immunohistochemistry using a avidin-biotin-peroxidase procedure followed by 3,3'diaminobenzidine (DAB) staining. SHAM and experimental tissues were always processed together to avoid confounding by slight differences in staining.

\section{ISH}

The primers were synthesized by Sigma-Aldrich with forward ' 5 ACCCCCTGCTATGTGTCTCA' 3 and reverse '5 TCACTGGAGCCTGAAAGTGC ' 3 for Per1 with a base length of $569 \mathrm{bp}$. The reverse primers were labeled in 5' with T7 polymerase promoter. An antisense DIG-RNA label was obtained using 200-ng purified PCR fragment for Per1, $2 \mu \mathrm{L}$ of DIG-RNA labeling mix (Roche), $2-\mu \mathrm{L}$ T7 RNA and polymerase (Roche), 2- $\mu \mathrm{L} 10 \times$ concentrated transcription buffer (Roche), and 40-U RNaseOUT (Invit- rogen), then supplemented with MilliQ diethyl pyrocarbonate (DEPC) water until $20 \mu \mathrm{L}$. Following incubation, the labeling efficiency of the DIG-labeling reaction was determined in a spot assay. The RNA dilutions were spotted on Nylon Membrane (Zeta probe, Bio-Rad), incubated with anti-DIG POD (Roche) and developed with DAB. The RNA was compared with labeled control RNA (Roche).

Following a short rinse in PBS, sections were fixed in $4 \%$ paraformaldehyde in PBS for 5 min and treated with $\mathrm{H}_{2} \mathrm{O}_{2} 3 \%$ in PBS-DEPC for 30 min. Sections were washed three times for $5 \mathrm{~min}$ in PBS and incubated in $0.5-\mu \mathrm{g} / \mathrm{mL}$ proteinase $\mathrm{K}$ in PBS with Triton $\mathrm{X}-1000.1 \%$ for $10 \mathrm{~min}$ at room temperature followed by 10 min postfixation in $4 \%$ paraformaldehyde in PBS.

Following washing and incubation in hibridization mix, overnight sections were rinsed and incubated with blocking solution 1\% (Roche) in buffer 1 for 30 min to block nonspecific protein binding. Sections were washed and the signal was amplified with TSA plus biotin kit (PerkinElmer) according to instructions. Finally, sections were incubated with Streptavidin HRP (The Jackson Laboratory) 1:500 in buffer 1 for 30 min. Following three washes in buffer 1, the reaction was revealed with DAB-Nickel, and sections were mounted with Entelan.

\section{RT-qPCR}

Total RNA was extracted from the liver using TRIzol reagent (life technologies) according to the manufacturer's protocol. The concentration and RNA quality were confirmed with agarose gel . Total RNA (2500 ng) from the liver was reverse transcribed to single strand cDNA using SuperScript III first-strand synthesis (Invitrogen) according to the manufacturer's protocol. The cDNA samples were quantified in Nanodrop and diluted in RNase free water at $250 \mathrm{ng} / \mu \mathrm{l}$ and stored at $-20^{\circ} \mathrm{C}$. For absolute quantification, we generated a standard calibration curve using highly purified standards that had been carefully quantified as to the amount or copy number. A standard curve was derived from setting out the threshold cycle against the number of RNA copies. The corresponding curves and logarithmic regression formulas were generated to replace necessary data obtained experimentally. Amplification was performed as follows: $1 \mu$ l of the first strand cDNA sample was mixed with 5- $\mu$ l SYBR select master mix (Applied biosystems), 2- $\mu$ l milliQ water, and $1-\mu \mathrm{l}$ primers mix 10mM (Sigma). A StepOne real-time PCR system (Applied biosystems) was used to amplify the genes from each liver sample in triplicate on a 48-well reaction plate using the following protocol: 10 -min denaturation at $95^{\circ} \mathrm{C}, 40$ cycles of $15 \mathrm{~s}$ denaturation at $95^{\circ} \mathrm{C}$ and $60 \mathrm{~s}$ annealing and extension at $60^{\circ} \mathrm{C}$. The sequences of primers used (designed by Sigma's OligoArchitect program): forward '5 ACATTCCTAACACAACCAA '3 and reverse ' 5 TGCTTGTCATCATCAGAG '3 for Per1; forward ' 5 CCTACTCTGATAGTTCGTCTA ' 3 and reverse '5 ATCCT TGGTCGTTGTCTA ' 3 for Bmal1; forward '5 GGAGAC TATATTAGGCGTTA ' 3 and reverse ' 5 CCTTCTGGATAC CTTCTG '3 for Cry1. 


\section{Quantification}

Pictures were taken by using an Axioplan microscope (Zeiss) equipped with a digital color camera (Olympus DP25, Olympus). The SCN and ARC were manually outlined; the Fos-positive nuclear profiles were automatically detected by means of size and staining threshold detection. The same parameters were used for all experimental groups using ImageJ software $(\mathrm{NIH})$. For each rat, two to three sections were measured $\sim 90 \mu \mathrm{m}$ apart; the mean number of c-Fospositive nuclear profiles from both SCN per section was calculated. ARC Per1-immunoreactivity (Per1-ir) was quantified in the same manner. Demarcating the ARC neuroanatomical landmarks were used to draw a triangular form in accordance to the rat brain atlas (Paxinos and Watson), also determining the equivalent sections between animals. All c-Fos or Per-1-ir positive nuclei were counted in this area. For SCN Per1 in situ quantification, a manual outline of the SCN was made, the mean optic density for that area was determined and the background optic density just outside the SCN was subtracted. For each rat, three sections were taken $\sim 90 \mu \mathrm{m}$ apart. Only the sections with the highest Per1 expression were analyzed and compared.

\section{Statistics}

All data were normally distributed and are expressed in mean \pm SEM. Statistical comparisons were performed using GraphPad Prism (GraphPad software). Data were analyzed using one-way ANOVA when appropriate. Analyzing SCN and ARC neuronal activity in different metabolic conditions a two-way ANOVA was used for factor group, i.e., SHAM versus RC cut (two levels) and factor metabolic condition, i.e., ad libitum, fasting, and glucose (three levels). This was followed by a Tukey's multicomparison post hoc test; $p<0.05$ was deemed significant. $\chi^{2} \mathrm{~d}\left(\chi^{2}\right)$ locomotor activity analysis was performed using SPAD9 (a program linked to our activity monitoring system).

A repeated measures (RM)-ANOVA was used for temperature and clock gene analysis with the factor group, i.e., SHAM versus $R C$ cut (two levels) and time as a factor of RM (23 levels for temperature and four levels for clock genes). For corticosterone and melatonin, the factor group, i.e., SHAM versus RC-cut versus SCNxARCx (three levels), was also set against time as a factor of RM (four levels). For the RM-ANOVA, $p<0.05$ was considered statistically significant. If a significant effect was reached, linear harmonic regression analysis was performed to determine circadian rhythmicity. CircWave version 1.4 software (Oster et al., 2006) was used employing an $F$ test to validate rhythmicity with an assumed period of $24 \mathrm{~h}$ and a threshold value of 0.05 for $\alpha$. For data visualization purposes where a significant rhythm in expression was detected, data were fitted with a sine wave function using GraphPad software.

\section{Results}

\section{SCN-ARC axis disruption causes arrhythmicity in DD conditions}

Animals kept in DD following bilateral ARC lesions demonstrated a clear loss of rhythm in locomotor activity (data not shown). Since these animals maintained a perfect rhythm under LD conditions we hypothesized that in the absence of light, the SCN requires an additional synchronizing stimulus from the ARC to drive its output. To investigate this hypothesis, coronal retrochiasmatic knife cuts (RC cuts) were made, leaving both ARC and SCN intact but severing their direct neuronal connections. $\chi^{2} d$ periodogram analysis of locomotor activity in light/dark (LD 12 h lights on/off, $7 \mathrm{~d}, n=7$ ), showed a significant diurnal rhythm in both SHAM and RC-cut animals; however, in DD (7 d, $n=7)$, all RC-cut animals were rendered arrhythmic (Fig. 2; Table 1). When RC cuts just missed the medial, most ventral part of the retrochiasmatic area, various phenotypes of rhythmicity could be observed during DD. Only animals showing arrhythmic locomotor activity in DD and in which subsequent anatomic analysis demonstrated the knife cut to be placed medially, in the retrochiasmatic area, were included for further analysis.

Temperature analysis showed a robust diurnal rhythm in LD in SHAM-operated (RM-ANOVA, $F_{(47,288)}=8.01909$, $p<0.0001 ; F$ test, $p<0.0001)$ and RC-cut animals $\left(F_{(47,288)}=4.7408, p<0.0001 ; F\right.$ test, $\left.p<0.0001\right)$. However, in DD RC-cut animals, failed to demonstrate a significant difference between time points $\left(F_{(47,288)}=1.195\right.$, $p=0.1925)$ as compared with SHAM animals $\left(F_{(47,288)}=\right.$ 4.492, $p<0.0001$; $F$ test, $p<0.0001$; Fig. 2C; Table 1). Thus, the pronounced rhythm seen during LD conditions, in contrast to the absent rhythms in DD, raises the question whether all functional SCN output was disrupted in DD and rescued in LD. Therefore, two to three weeks after surgery, corticosterone and melatonin levels were analyzed from tail blood samples at ZT0, ZT6, ZT12, and ZT18. Surprisingly, despite their rhythmic temperature and locomotor activity in LD, animals with an SCN-ARC $\mathrm{RC}$ cut showed no significant variation among different time points in corticosterone levels during LD (RM-ANOVA, $F_{(3,22)}=$ $1.707, p=0.1947)$, contrary to $\operatorname{SHAM}\left(F_{(3,21)}=25.45, p<\right.$ 0.0001 ; Fig. $3 A)$ with a peak at ZT12 $(p<0.0001)$. However, the same animals did express a significant effect of time in melatonin levels $\left(F_{(3,19)}=7.219, p=0.002\right)$ similar to $\operatorname{SHAM}\left(F_{(3,16)}=15.47, p<0.0001 ;\right.$ Fig. $\left.3 A\right)$ with a peak at ZT18 $(p=0.0025$ ). Blood samples collected $36 \mathrm{~h}$ following DD at CT0, CT6, CT12, and CT18 demonstrated that melatonin, despite absent temperature and activity rhythms, continued to be rhythmic in DD in both SHAM $\left(F_{(3,13)}=21.04, p<0.0001\right)$ and RC-cut animals $\left(F_{(3,12)}=\right.$ $4.665, p=0.022$; Fig. $3 A)$ with a peak at CT18 $(p=$ $0.0221)$. Considering that corticosterone was significantly arrhythmic in LD, duplicate measurements in DD were not regarded as additive. These observations demonstrate that, although the majority of the determined physiologic rhythms were absent in DD conditions, melatonin secretion occurs independent of the SCN-ARC interaction. Furthermore, the present results show that corticosterone rhythm, known to depend on the integrity of the SCN, also depends on the integrity of SCN-ARC communication.

\section{Interaction between the SCN and ARC is essential for rhythmicity}

To confirm that only the interaction between the SCN and ARC was essential for rhythmicity and the observed 
A

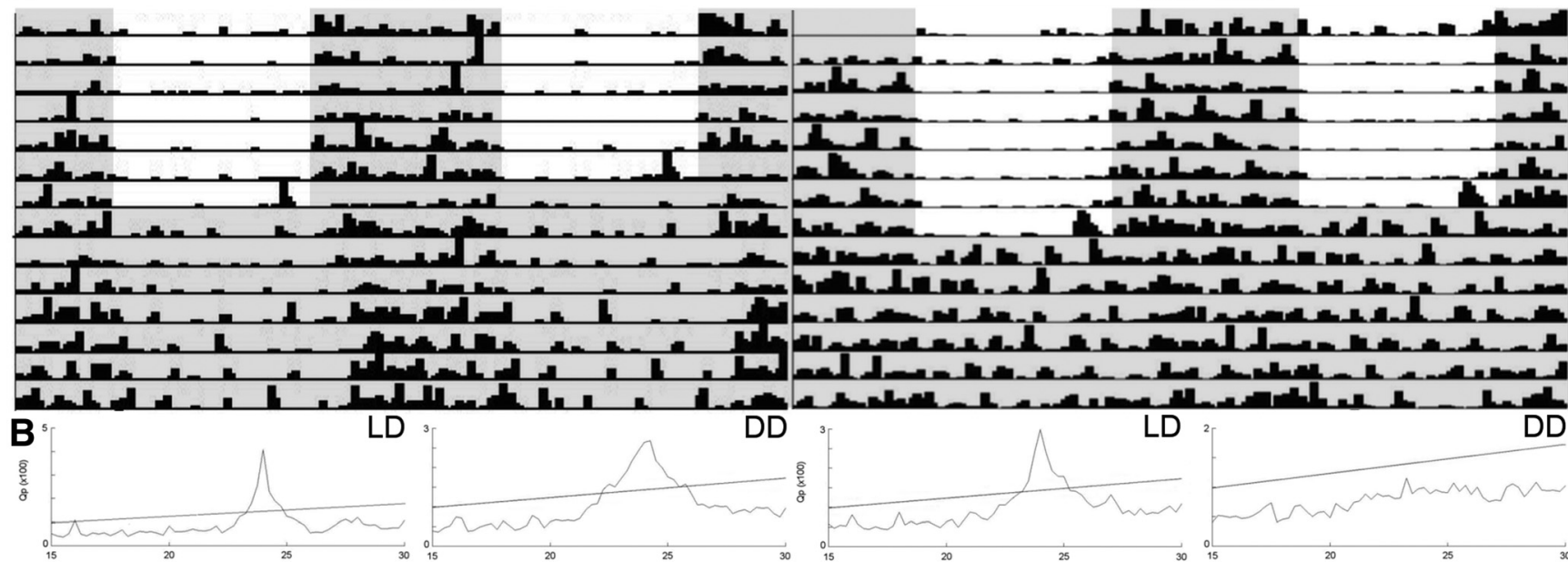

C
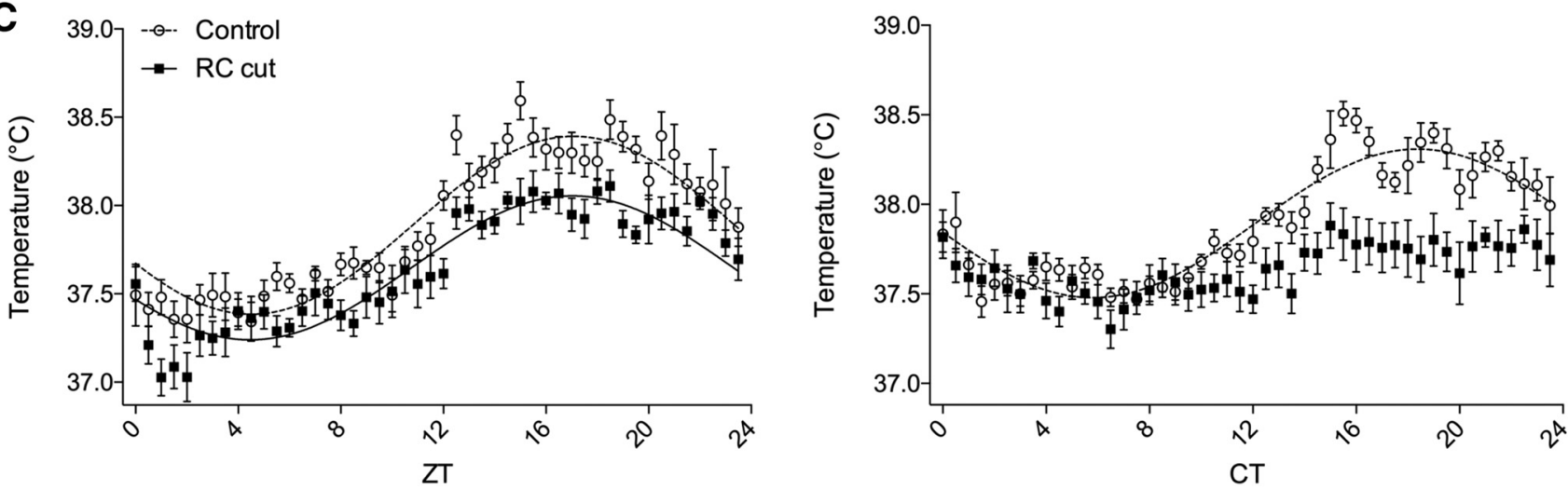

Figure 2. Arrhythmic locomotor activity and temperature observed in DD following retrochiasmatic knife cuts (RC cut). $\boldsymbol{A}$, Representative actograms of a SHAM-operated animal (left) and an RC-cut animal (right), in LD and DD conditions (shading indicates lights off). Following RC cuts, animals recovered in LD and showed normal diurnal rhythmicity, subsequent DD conditions rendered all $\mathrm{RC}$-cut animals arrhythmic. $\boldsymbol{B}, \chi^{2}$ periodogram analysis of LD and DD periods of SHAM (left) and RC cut (right) animals. The slanted line in the periodogram indicates $p=0.01$. C, For RC-cut animals temperature was arrhythmic in DD. Note the strong temperature decrease in RC-cut animals between ZT0 and ZT2 in reaction to light during LD. The graph is a double plot of temperature data in SHAM and RC-cut animals in LD (left) and DD (right). Each value represents seven animals (mean \pm SEM). Only where a significant rhythm in expression was detected, data were fitted with a sine wave function $(p<0.05, F$ test).

arrhythmicity was not simply caused by damage to the retrochiasmatic area, we made unilateral electrolytic SCN lesions in combination with contralateral $90^{\circ}$ retrochiasmatic knife cuts. Thus, maintaining the $A R C$, the unilateral SCN and unilateral retrochiasmatic area intact, while also the unilateral afferents and efferents of ARC and retrochiasmatic area were not disrupted, the reciprocal communication between SCN and ARC was still effectively removed (Fig. 1B,D). Contrary to unilateral SCN or mediobasal hypothalamus lesions, shown to be ineffective in

Table 1. Analysis of activity and temperature rhythms in SHAM, RC-cut, and $S_{C} N_{X} A R C$ Cut animals during LD and DD conditions

\begin{tabular}{|c|c|c|c|c|c|c|c|}
\hline & & LD & & & $\mathrm{DD}$ & & \\
\hline Control & $\mathrm{RC}$ cut & $\mathrm{SCN}_{X} \mathrm{ARC}^{\text {Cut }}$ & Control & $\mathrm{RC}$ cut & $\mathrm{SCN}_{X} \mathrm{ARC}^{\text {Cut }}$ & & \\
\hline \multirow[t]{3}{*}{ Activity } & Acrophase (ZT/CT) & $17.23 \pm 0.4$ & $16.6 \pm 0.55$ & $15.93 \pm 0.38$ & $17.88 \pm 0.63$ & - & - \\
\hline & Amplitude & $1.79 \pm 0.15$ & $1.68 \pm 0.22$ & $1.42 \pm 0.12$ & $1.36 \pm 0.07 \#$ & - & - \\
\hline & $R^{2}$ & 0.5 & 0.45 & 0.5 & 0.42 & 0.05 & 0.12 \\
\hline Rhythmic & $7 / 7$ & $7 / 7$ & $6 / 6$ & $7 / 7$ & $0 / 7$ & $0 / 6$ & \\
\hline \multirow[t]{4}{*}{ Temperature } & Mean $\left({ }^{\circ} \mathrm{C}\right)$ & $37.43 \pm 0.04$ & $37.66 \pm 0.06 *$ & $37.77 \pm 0.05 * *$ & $37.43 \pm 0.04$ & $37.63 \pm 0.0 .07$ & $37.79 \pm 0.02 * *$ \\
\hline & Acrophase (ZT/CT) & $16.9 \pm 0.36$ & $16.95 \pm 0.56$ & $16.06 \pm 0.42$ & $17.99 \pm 0.29$ & - & - \\
\hline & Amplitude & $0.49 \pm 0.032$ & $0.38 \pm 0.04$ & $0.33 \pm 0.03$ & $0.42 \pm 0.04$ & - & - \\
\hline & $R^{2}$ & 0.67 & 0.59 & 0.54 & 0.59 & 0.14 & 0.23 \\
\hline Rhythmic & $7 / 7$ & $7 / 7$ & $4 / 4$ & $7 / 7$ & $1 / 7$ & $0 / 4$ & \\
\hline
\end{tabular}


Table 2. Statistical table

\begin{tabular}{|c|c|c|c|c|}
\hline Figure & Panel & Distribution & Test & $p$ value \\
\hline \multirow[t]{4}{*}{2} & $\mathrm{Ca}$ & Normal distribution & $\begin{array}{l}\text { RM-ANOVA } \\
F \text { test }\end{array}$ & $\begin{array}{l}F_{(47,288)}=8.01909, p<0.0001 \\
p<0.0001\end{array}$ \\
\hline & $\mathrm{Ca}$ & Normal distribution & $\begin{array}{l}\text { RM-ANOVA } \\
F \text { test }\end{array}$ & $\begin{array}{l}F_{(47,288)}=4.7408, p<0.0001 \\
p<0.0001\end{array}$ \\
\hline & $\mathrm{Cb}$ & Normal distribution & $\begin{array}{l}\text { RM-ANOVA } \\
F \text { test }\end{array}$ & $\begin{array}{l}F_{(47,288)}=4.492, p<0.0001 \\
p<0.0001\end{array}$ \\
\hline & $\mathrm{Cb}$ & Normal distribution & RM-ANOVA & $F_{(47,288)}=1.195, p=0.1925$ \\
\hline \multirow[t]{8}{*}{3} & $\mathrm{Aa}$ & Normal distribution & RM-ANOVA & $F_{(3,21)}=25.45, p<0.0001$ \\
\hline & $\mathrm{Aa}$ & Normal distribution & RM-ANOVA & $F_{(3,22)}=1.707, p=0.1947$ \\
\hline & $\mathrm{Aa}$ & Normal distribution & RM-ANOVA & $F_{(3,17)}=1.048, p=0.3967$ \\
\hline & $\mathrm{Ab}$ & Normal distribution & RM-ANOVA & $F_{(3,16)}=15.47, p<0.0001$ \\
\hline & $A b$ & Normal distribution & RM-ANOVA & $F_{(3,19)}=7.219, p=0.002$ \\
\hline & $\mathrm{Ab}$ & Normal distribution & RM-ANOVA & $F_{(3,11)}=26.38, p<0.0001$ \\
\hline & $A c$ & Normal distribution & RM-ANOVA & $F_{(3,13)}^{(3,11)}=21.04, p<0.0001$ \\
\hline & Ac & Normal distribution & RM-ANOVA & $F_{(3,12)}=4.665, p=0.022$ \\
\hline \multirow[t]{2}{*}{3} & $\mathrm{Ca}$ & Normal distribution & $\begin{array}{l}\text { RM-ANOVA } \\
F \text { test }\end{array}$ & $\begin{array}{l}F_{(3,16)}=0.9766, p=0.4283 \\
F_{(3,16)}=15.23, p<0.0001 \\
F_{(1,16)}=4.188, p=0.0575 \\
p=0.0099 / p=0.0123\end{array}$ \\
\hline & $\mathrm{Cb}$ & Normal distribution & $\begin{array}{l}\text { RM-ANOVA } \\
F \text { test }\end{array}$ & $\begin{array}{l}F_{(3,16)}=11.14, p=0.0003 \\
F_{(3,16)}=11.80, p=0.0003 \\
F_{(1,10)}=6.136, p=0.0248 \\
p=0.01305 / p=0.2178\end{array}$ \\
\hline \multirow[t]{3}{*}{4} & A & Normal distribution & $\begin{array}{l}\text { RM-ANOVA } \\
F \text { test }\end{array}$ & $\begin{array}{l}F_{(3,8)}=4.43, p=0.0410 / F_{(3,10)}=8.53, p=0.0041 \\
p=0.0254 / p=0.0038\end{array}$ \\
\hline & B & Normal distribution & $\begin{array}{l}\text { RM-ANOVA } \\
F \text { test }\end{array}$ & $\begin{array}{l}F_{(3,8)}=21.93, p=0.0003 / F_{(3,10)}=2.56, p=0.1134 \\
p \stackrel{0}{=} 0.0043 / p=0.268\end{array}$ \\
\hline & $\mathrm{C}$ & Normal distribution & $\begin{array}{l}\text { RM-ANOVA } \\
F \text { test }\end{array}$ & $\begin{array}{l}F_{(3,8)}=8.009, p=0.0086 / F_{(3,10)}=2.373, p=0.1316 \\
p=0.0023 / p=0.1372\end{array}$ \\
\hline 5 & $\mathrm{Ca}$ & Normal distribution & 2ANOVA & $\begin{array}{l}F_{(2,25)}=3.489, p=0.0461 \\
F_{(2,25)}=9.341, p=0.0009 \\
F_{(1,25)}=93.09, p<0.0001\end{array}$ \\
\hline 5 & $\mathrm{Cb}$ & Normal distribution & 2ANOVA & $\begin{array}{l}F_{(2,25)}=15.59, p<0.0001 \\
F_{(2,25)}=2.838, p=0.0775 \\
F_{(1,25)}=3.6, p=0.0694\end{array}$ \\
\hline
\end{tabular}

RM-ANOVA, ANOVA (one-way or two-way); 2ANOVA, two-way ANOVA; $F$ test, linear harmonic regression analysis.

changing rhythmicity (Gerkema et al., 1990), our unilateral SCN-lesion combined with contralateral ARC-isolation $\left(\mathrm{SCN}_{\mathrm{X}} \mathrm{ARC} \mathrm{Cut}^{\mathrm{Cu}}\right.$ ) animals were behaviorally arrhythmic in $\mathrm{DD}$ as was temperature (Table 1). As with RC-cut animals, $\mathrm{SCN}_{\mathrm{X}} \mathrm{ARC} \mathrm{Cut}^{\mathrm{Cu}}$ animals showed arrhythmic corticosterone $\left(F_{(3,17)}=1.048, p=0.3967\right)$ but rhythmic melatonin levels in LD $\left(F_{(3,11)}=26.38, p<0.0001\right.$; Fig. $\left.3 A\right)$ according to ANOVA analysis (see details on statistical analysis table 2). This confirmed that the observed arrhythmicity in RCcut animals was indeed due to elimination of SCN-ARC intercommunication.

\section{The SCN remains rhythmic following SCN-ARC deafferentation}

Observing the circadian disruption of RC-cut animals in DD, we hypothesized that either (1) specific SCN output is altered, with the SCN depending on synchronized concomitant ARC output for adequate regulation of physiologic function; or (2) the robustness of SCN rhythmicity is affected, resulting in diminished clock function and circadian control, only revealed during DD conditions. We analyzed SCN Per1 expression in DD conditions through ISH with animals killed $36 \mathrm{~h}$ after lights off at CT0, CT6, CT12, and CT18. RC-cut animals showed a slightly higher expression of Per1 at all time points, but with equal amplitude of Per1 expression as compared with SHAM animals (RM-ANOVA, $F_{(3,16)}=0.9766, p=0.4283$; Fig. $3 C)$. Also, a preserved Per1 circadian rhythmicity in both $\operatorname{SHAM}\left(F_{(3,16)}=15.23, p<0.0001 ; F\right.$ test, $\left.p=0.0099\right)$ and RC-cut animals ( $F$ test, $p=0.0123$ ) was found. This demonstrates SCN rhythmicity is not significantly affected by SCN-ARC axis disruption.

Next, we hypothesized that the rhythmic SCN, together with rhythmic melatonin levels, implies an intact autonomic control over peripheral clock gene rhythmicity, though to some extent other physiologic processes like corticosterone rhythm and food intake also have their impact on peripheral clock-gene rhythmicity. We measured clock gene expression in the liver of SHAM and experimental animals using RT-qPCR. Per1 showed a clear circadian oscillation in both SHAM (RM-ANOVA, $F_{(3,8)}=4.43, p=0.0410 ; F$ test, $p=$ $0.0254)$ and RC-cut animals $\left(F_{(3,10)}=8.53, p=0.0041\right.$; $F$ test, $p=0.0038)$. However, Bmal1 failed to show an effect of time $\left(F_{(3,10)}=2.56, p=0.1134 ; F\right.$ test, $\left.p=0.268\right)$ in experimental animals as compared with SHAMs $\left(F_{(3,8)}=\right.$ 21.93, $p=0.0003$; $F$ test, $p=0.0043)$; likewise, Cry1 expression in experimental animals showed no effect of time, nor circadian rhythmicity $\left(F_{(3,10)}=2.373, p=0.1316 ; F\right.$ 

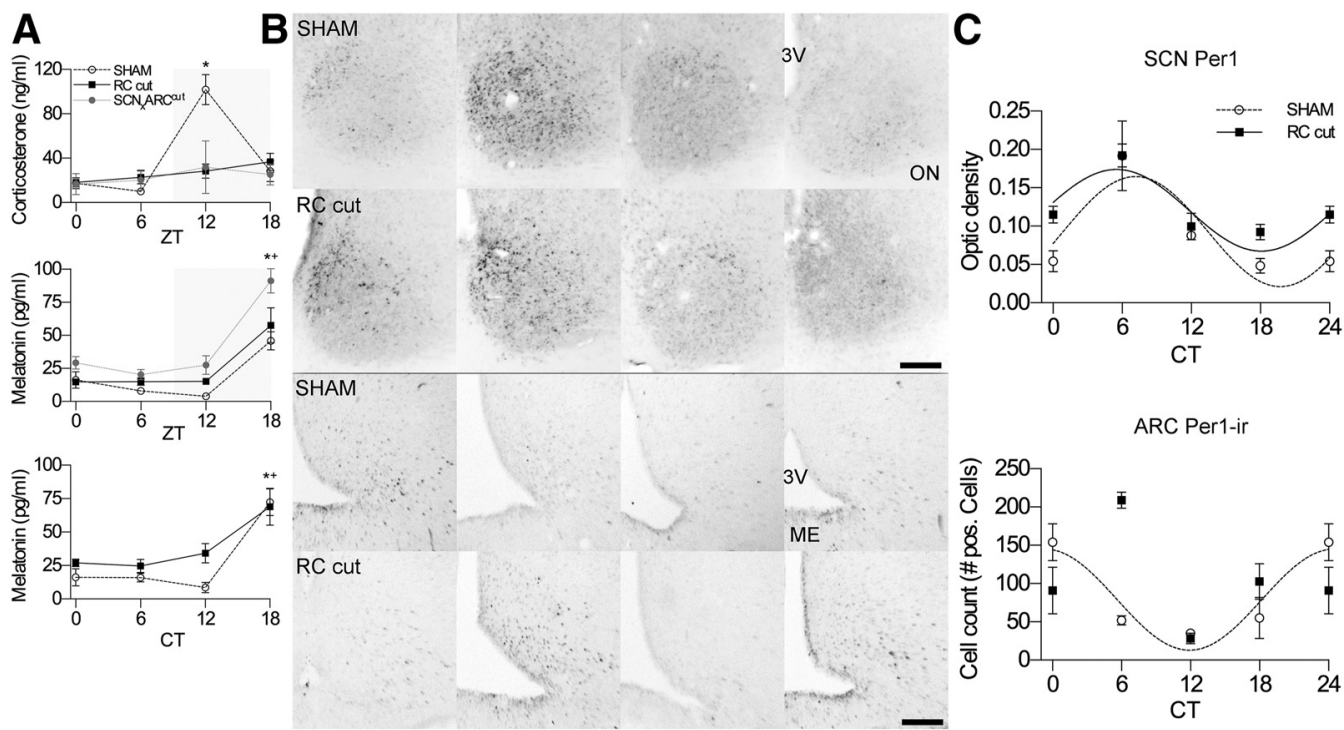

0

CT

12

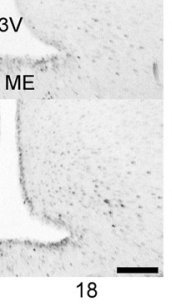

Figure 3. In RC-cut animals corticosterone rhythm was lost while melatonin remained intact similar to SCN rhythmicity. Rhythm of ARC Per1 expression was attenuated, demonstrating SCN-ARC desynchronization. $\boldsymbol{A}$, Corticosterone levels showed a significant peak at ZT12 in SHAM but not RC-cut or SCN ARC $^{\text {cut }}$ animals, indicating loss of rhythmicity. Melatonin maintained its rhythm in LD and DD in both SHAM and experimental animals. Data represents $N=5-7$, and $N=4-6$ for the $\mathrm{SCN}_{\mathrm{X}} \mathrm{ARC}{ }^{\mathrm{Cut}}$ group (mean $\pm \mathrm{SEM}$ ). $* p<0.05(\mathrm{SHAM})$, $+p<0.05$ (Experimental), ANOVA analysis. B, Representative photomicrographs of SCN Per1 mRNA expression (above) and ARC Per1-ir (below) in SHAM and RC-cut animals. Scale bar, $90 \mu \mathrm{m}$. 3V, third ventricle; ON, optic nerve; ME, median eminence. C, Per1 mRNA analysis of the SCN demonstrates significant circadian rhythmicity in RC-cut $(p<0.05)$ and in SHAM animals $(p<0.01)$. The ARC failed to show a significant rhythm in RC-cut animals $(p>0.05)$ as compared with SHAM. The higher point at CT6 suggests a phase advance; however, an $F$ test failed to show a significant circadian rhythm. Data represents $N=3-4$ with a double-plot of CT0 at CT24. Only where a significant rhythm in expression was detected, data were fitted with a sine wave function ( $p<0.05, F$ test).

test, $p=0.1372)$ unlike in SHAMs $\left(F_{(3,8)}=8.009, p=\right.$ 0.0086; $F$ test, $p=0.0023$; Fig. 4).

\section{The SCN and ARC desynchronize}

Under normal conditions Per1 expression in the ARC shows a diurnal rhythm in vivo (Shieh et al., 2005) with a peak around ZT18. To investigate whether the known rhythm of Per1 protein in the ARC would depend on neuronal interaction with the SCN we also investigated Per1 protein expression in the ARC after SCN deafferentation through $\mathrm{RC}$ cuts. This showed a significant difference between SHAM and RC-cut animals (RM-ANOVA, $\left.F_{(1,10)}=6.136, p=0.0248\right)$, a significant effect of time
$\left(F_{(3,16)}=11.14, p=0.0003\right)$ but also a significantly different time trend in ARC PER1 expression between SHAM en RC-cut animals $\left(F_{(3,16)}=11.80, p=0.0003\right)$. Testing for circadian rhythmicity revealed that SHAM animals showed a clear rhythm ( $F$ test, $p=0.01305$ ) with peak expression around CT23 (22.98 \pm 0.73$)$; however, in experimental animals PER1 failed to express circadian rhythmicity in the ARC ( $F$ test, $p=0.2178$; Fig. $3 C$ ). Together with the preserved circadian Per1 expression in the SCN, this indicates a significant desynchrony between SCN and ARC in RC-cut animals as compared with SHAM animals.
Per1

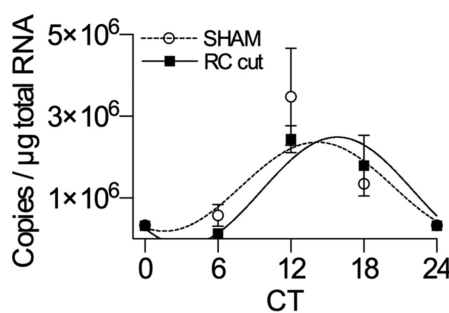

Bmal1

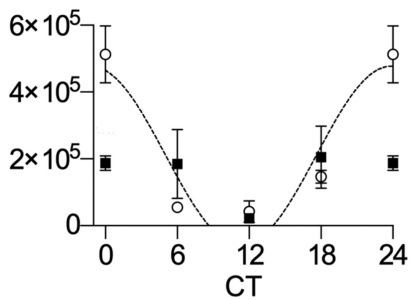

Cry1

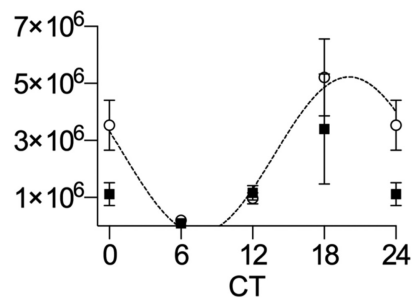

Figure 4. Peripheral liver clock genes in experimental animals show contrasting changes in rhythmicity as compared with SHAM animals. Per1 gene expression in experimental animals showed no significant changes in rhythmicity $(p<0.01)$ as compared with SHAM $(p<0.05)$, contrary to Bmal1 $(p=0.268)$ and Cry1 $(p=0.137)$ failing to demonstrate rhythmicity as compared with SHAM animals $(p<0.05)$. Each value represents three to four animals (mean \pm SEM). CT24 is a double-plot of CTO. Solid squares indicate $\mathrm{RC}$-cut animals and open circles SHAM animals. Only where a significant rhythm in expression was detected, data were fitted with a sine wave function $(p<0.05, F$ test). 

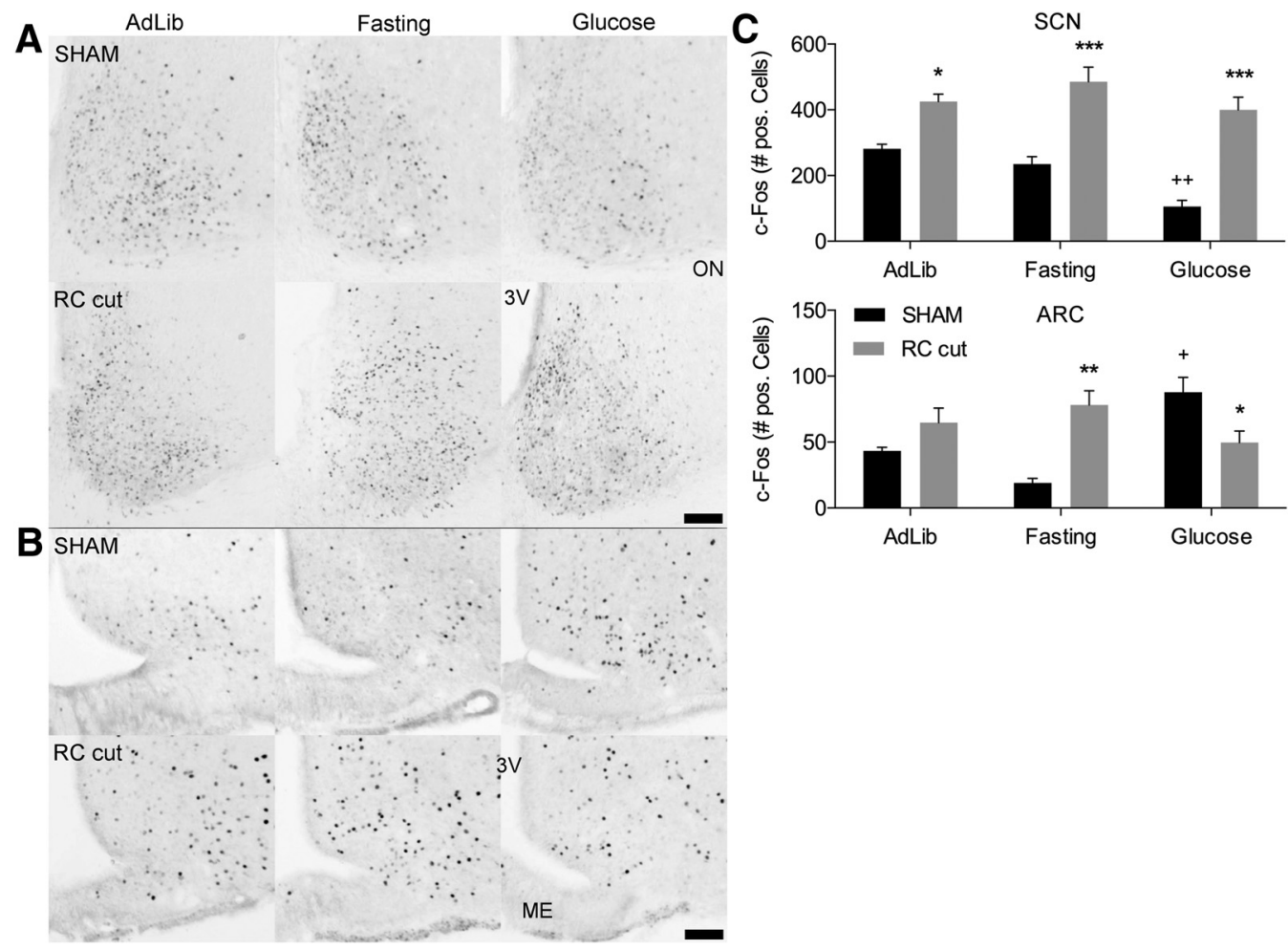

Figure 5. c-Fos staining of the SCN and ARC showing significant changes in Fos expression following glucose intake in SHAM animals, whereas experimental animals show no significant alterations in Fos expression. $\boldsymbol{A}$, Representative photomicrographs of SCN c-Fos expression in SHAM en RC-cut animals in ad libitum, fasting or fasting and glucose intake. B, ARC c-Fos expression in SHAM en RC-cut animals during above stated conditions. $\boldsymbol{C}$, Bar charts of c-Fos quantification in the SCN and ARC of SHAM en $\mathrm{RC}$-cut animals. All animals were killed at ZT4 following ad libitum, $48 \mathrm{~h}$ of fasting or fasting followed by 5 -ml $3 \%$ glucose intake 2 h before killing. Each value in $\boldsymbol{C}$ represents three to four animals (mean \pm SEM). Scale bar, $70 \mu \mathrm{m} ; *$ significant between group difference; +significant within group difference. $* /+p<0.05, * * /++p<0.01$, $* * * /+++p<0.001$ (ANOVA analysis).

\section{RC cuts reveal altered metabolic SCN-ARC communication}

To investigate whether ARC-SCN metabolic communication, was impaired by the RC cuts, we subjected animals to a protocol of fasting followed by a brief glucose stimulus. Two-way ANOVA analysis of c-Fos expression in the SCN revealed a significantly different reaction to treatment (ad libitum, fasting, and glucose) between SHAM en RC-cut animals $\left(F_{(2,25)}=3.489, p=0.0461\right.$; Fig. 5). SHAM animals drinking $5 \mathrm{ml}$ of a $3 \%$ glucose solution following $48 \mathrm{~h}$ of fasting showed a strong decrease in c-Fos expression in the SCN $(p<0.01)$ as compared with ad libitum conditions. Conversely, RC-cut animals showed no significant change in c-Fos expression in the $\operatorname{SCN}(p>0.05$; Fig. 5). Also, merely fasting, known to result in a moderate decrease of c-Fos in the ventral SCN (Saderi et al., 2013) did not result in any change in the RC-cut animals. Furthermore, a comparison of SHAM and RC-cut animals showed a significantly elevated SCN c-Fos expression in RC-cut animals in all conditions as compared with SHAM animals.

Also in the ARC, c-Fos expression in reaction to ad libitum, fasting, or glucose was significantly different in RC-cut animals as compared with SHAM animals $\left(F_{(2,25)}=15.59\right.$, $p<0.0001)$. In reaction to the glucose stimulus, the ARC in SHAM animals showed a significant increase in c-Fos $(p<0.01)$ as compared with ad libitum conditions, while
RC-cut animals, again, demonstrated no significant difference between conditions $(p=0.0775)$. Thus, SHAM animals showed a decrease in SCN c-Fos activity that coincided with an increase of c-Fos in the ARC. This reciprocal influence is clearly lost in RC-cut animals, which showed no significant change in c-Fos expression in the SCN or ARC between different conditions.

\section{Deafferentation of SCN-ARC neuronal connections does not damage their efferents to other brain areas}

Since for most of our observations we used knife cuts in the retrochiasmatic area, we investigated whether this might have also severed other connections between the SCN and its target areas, other than the ARC. Also, we established whether knife cuts removed ARC output to efferent brain areas, especially dorsal and rostral of the cut. Immunohistochemical staining for VIP, recognized as one of the major output systems of the SCN (Takahashi, 1989), revealed that, except for its projections to the ARC, VIP innervation to the SPZ, PVN, and DMH remained unaffected (Fig. $6 A--C$ ). VIP innervation of the ARC and the area between the median eminence and ARC, present in SHAMs, disappeared in RC-cut animals (Fig. 6D,E). No statitical analysis was performed on the extent of loss or preservation of innervation since results were binary. Also NPY staining in the SCN reflecting input from the IGL (Fig. $6 F$ ) was not affected by RC cuts. Through unilateral ARC 


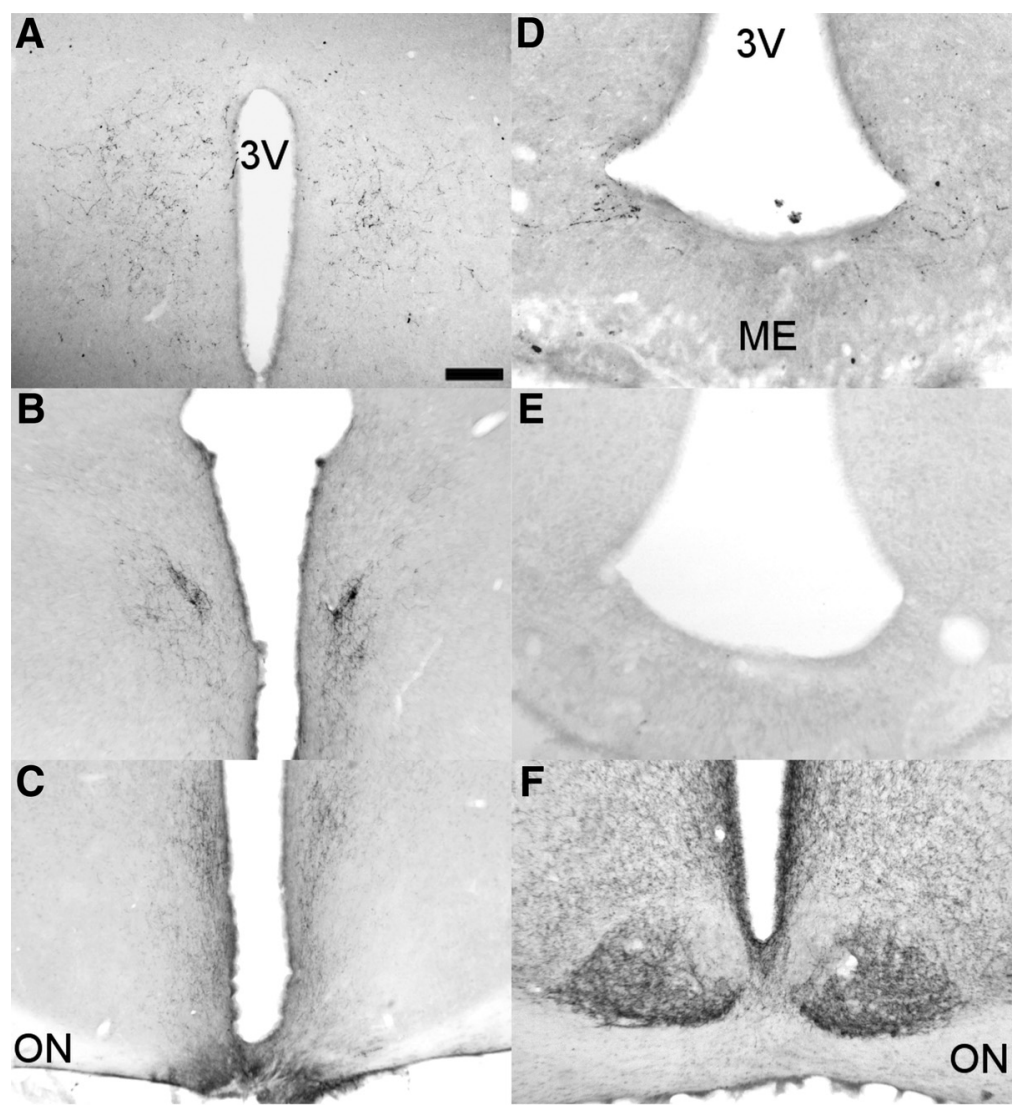

Figure 6. RC cuts do not affect main SCN output other than the ARC. Clear VIP innervation of the DMH $(\boldsymbol{A})$ and PVN $(\boldsymbol{B})$ in RC-cut animals. C, Also VIP staining to the SPZ and retrochiasmatic area dorsal to the knife cut is still intact in RC-cut animals. $\boldsymbol{D}$, Staining of VIP afferents in the ARC, clearly visible in SHAM animals, disappear following RC cut (E). $\boldsymbol{F}$, Likewise, NPY SCN staining, shown to be predominantly IGL derived, is not damaged by RC cuts. Pictures $\boldsymbol{A}-\boldsymbol{C}, \boldsymbol{E}, \boldsymbol{F}$ are from RC animals, only $\boldsymbol{D}$ shows staining in an intact animal. Scale bar, $130 \mu \mathrm{m}(\boldsymbol{A}$ and $\boldsymbol{F}), 175 \mu \mathrm{m}(\boldsymbol{B}), 250 \mu \mathrm{m}(\boldsymbol{C}), 70 \mu \mathrm{m}(\boldsymbol{D}$ and $\boldsymbol{E})$.

CtB injections in animals with ipsilateral $\mathrm{RC}$ cuts, we confirmed the loss of SCN connections with the ARC as compared with controls (data not shown).

Analysis of ARC-specific output by means of AgRP staining showed a normal distribution of AgRP in RC-cut animals in main ARC target areas, i.e., the POA, PVN, $\mathrm{VMH}$, and $\mathrm{DMH}$, suggesting normal ARC output unimpaired by the retrochiasmatic knife cut (Fig. 7). Assessing the completeness of SCN denervation from ARC efferents, AgRP staining showed a clear attenuation of SCN innervation in RC-cut animals compared with SHAMs (Fig. 8). Together with the unilateral SCN lesion and contralateral knife cut, these anatomic analyses demonstrate that reciprocal connections between the SCN and ARC are affected by our microcuts, but other SCN and ARC efferents remain intact.

\section{Discussion}

Most physiologic processes have well-defined circadian rhythms. The present study challenges the still prevalent view that these rhythms are driven solely by the $\mathrm{SCN}$. We demonstrate that generation of specific physiologic and behavioral circadian rhythms greatly depend on functional interaction between the SCN and ARC. RC cuts, effectively eliminating reciprocal communication between the SCN and ARC, resulted in a complete loss of rhythm in corticosterone secretion, whereas during DD conditions also locomotor activity and body temperature rhythms were completely lost. These findings are in agreement with prior studies demonstrating a role for the mediobasal hypothalamus in facilitating circadian rhythmicity (Gerkema et al., 1990) and consistent with observed loss of rhythm in adrenal corticosterone levels following knife cuts posterior to the SCN (Moore and Eichler, 1972). In the present study, we show that the reciprocal connectivity between SCN and ARC (SaebParsy et al., 2000; Yi et al., 2006) is essential to synchronize hormonal and behavioral rhythms.

\section{Interaction between SCN and ARC is essential for generating rhythms}

The striking observation that corticosterone rhythmicity was lost in RC-cut animals during LD conditions suggests a crucial role for the ARC in generating this rhythm jointly with the SCN. A recent observation that glucocorticoid receptors in the ARC are essential for the circadian variation in the negative feedback of circulating corticosterone on its own release (Leon-Mercado et al., 2017) supports this observation. Locomotor activity, rhythmic in LD, was completely arrhythmic in DD. One could argue that considering locomotor activity is rhythmic in LD conditions, for maintaining this rhythm in DD interaction be- 


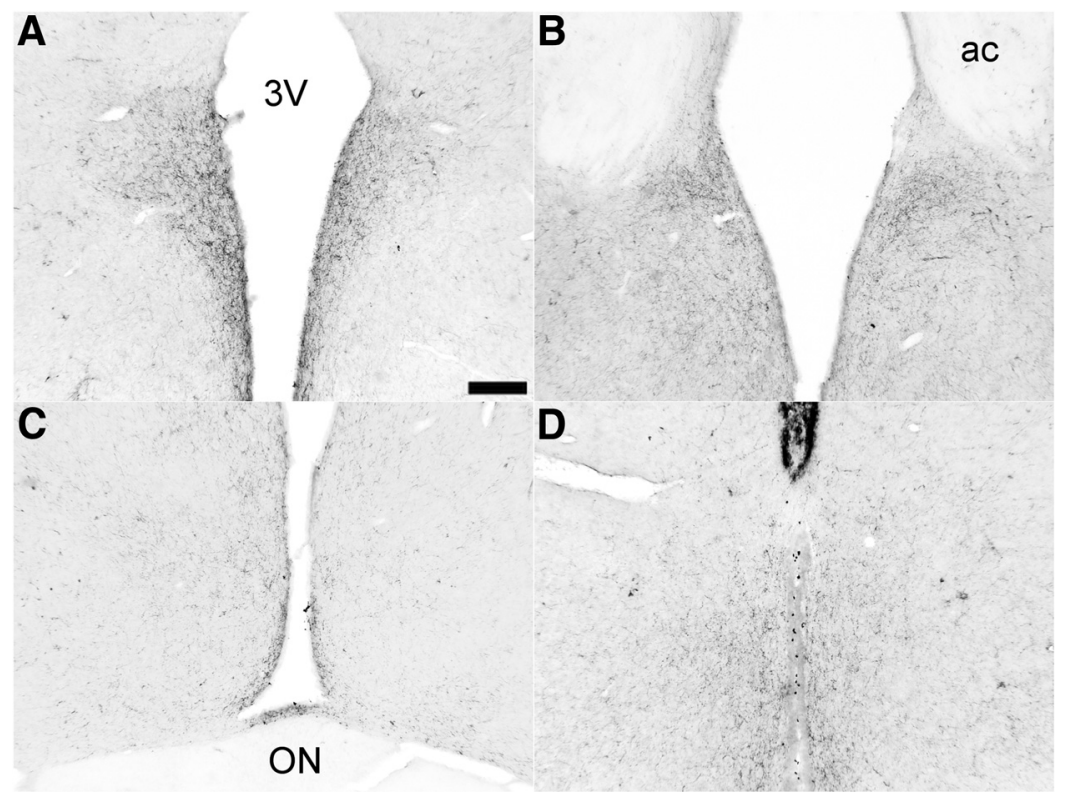

Figure 7. ARC-specific AgRP staining shows intact innervation of $(\boldsymbol{A})$ the PVN, (B) MnPO, (C) AVPV, and $(\boldsymbol{D})$ DMH, not affected by the knife cuts. Scale bar, $175 \mu \mathrm{m}(\boldsymbol{A}-\boldsymbol{C})$ and $215 \mu \mathrm{m}(\boldsymbol{D})$. All photomicrographs are from RC-cut animals. 3V, third ventricle; ON, optic nerve.

tween SCN and ARC is essential. On the other hand, there is the suggestion that the observed behavioral phenotype in LD is not truly rhythmic but rather shows diurnal variance due to the "masking" effect of light (Redlin and Mrosovsky, 1999). In view of the effect of locomotor activity on temperature, the rhythmic locomotor activity in LD in RC-cut animals may also be responsible for the observed rhythmic temperature in LD, while lost in DD. The present results show that for maintaining rhythmicity in $\mathrm{DD}$, interaction between SCN and ARC is essential.

The SCN receives numerous types of nonphotic feedback signals capable of altering the phase of the clock.
Activity can synchronize and phase-shift the SCN (Yamazaki et al., 1998; Schaap and Meijer, 2001), SCN clock genes can be synchronized to food in LL conditions (Lamont et al., 2005), while extra-SCN areas can influence the phase of SCN clock gene, Per1 (Vansteensel et al., 2003). Considering this, we hypothesized that severed SCN-ARC connections resulting in diminished nonphotic, metabolic feedback from the ARC to SCN (Yi et al., 2006) as well as aberrant rhythms in behavior, temperature, and corticosterone could lead to suppression of SCN rhythmicity. Yet, SCN rhythmicity measured by Per1 expression remained unaltered in RC-cut animals. This observation

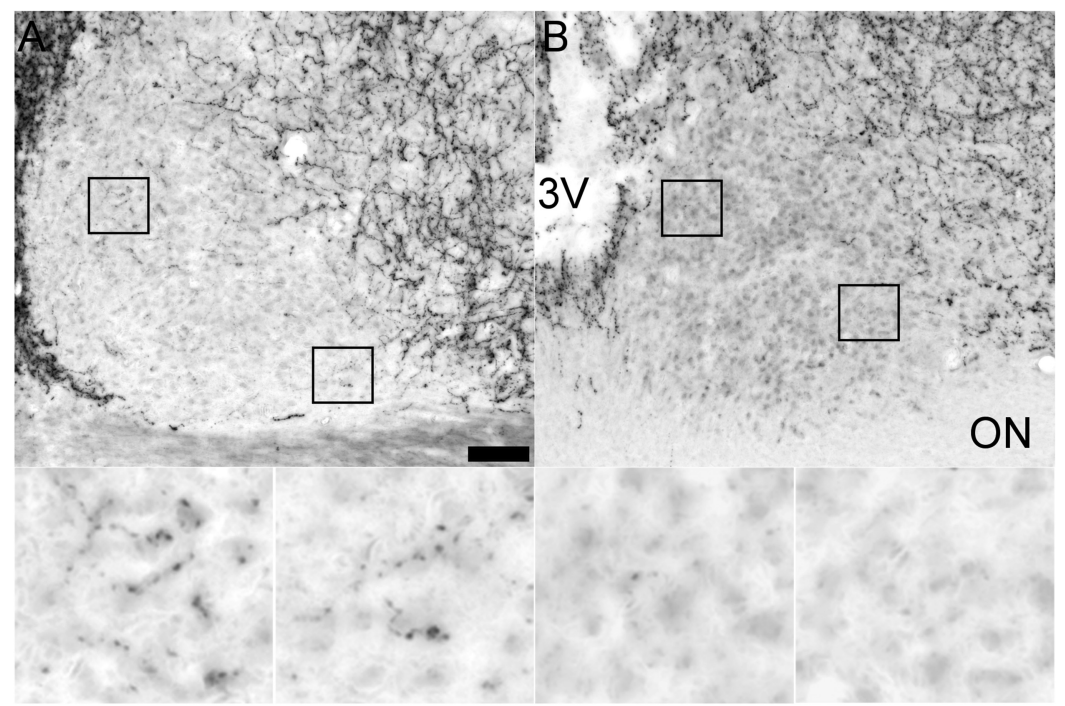

Figure 8. RC cuts strongly temper ARC innervation of the SCN as illustrated by diminished of ARC-specific AgRP staining. Representative photomicrographs of AgRP staining showing intact innervation of $(\boldsymbol{A})$ the SCN in a SHAM animal. $\boldsymbol{B}$, SCN of an RC-cut animal with strongly reduced AgRP innervation. Bottom pictures are magnifications of outlined boxes in $\boldsymbol{A}$ and $\boldsymbol{B}$ showing clear fibers in the SCN of control animals not seen in RC-cut animals. Scale bar, $70 \mu \mathrm{m}(\boldsymbol{A}$ and $\boldsymbol{B})$, and $15 \mu \mathrm{m}$ (the magnifications below). 3V, third ventricle; ON, optic nerve. 
suggests that, in the short term, the SCN is able to maintain its own autonomous rhythm in clock gene expression and that to drive physiologic functions, it depends on its interaction with the ARC. The observation that SCN-lesioned animals receiving SCN transplants from donor animals regain circadian rhythmicity, suggests that they do not require ARC interconnectivity. However, it has been shown that diffusible substances from the transplanted SCN into the third ventricle also restore locomotor activity rhythms (Silver et al., 1996). Since the $\mathrm{ARC}$, as a circumventricular organ, takes up substances from the third ventricle, it could be an effective target for SCN-derived diffusible substances.

Further assessing hypothalamic output through peripheral clock gene rhythmicity, we found liver Per1 expression of experimental animals to be similar to SHAM animals. However, no rhythm could be detected in liver Bmal1 and Cry1 clock gene expression. This anomaly could be explained by the fact that while there is no rhythm in corticosterone, activity, temperature, and possibly food intake ( $\mathrm{Li}$ et al., 2012), the persistent rhythm in melatonin could have its effect on liver Per 1 expression (Houdek et al., 2016).

\section{Circadian rhythms depend on synchronous SCN and ARC output}

A recent study has demonstrated that SCN driven activity of ARC $\alpha$-MSH neurons is essential for diurnal temperature control (Guzman-Ruiz et al., 2015). Moreover the study demonstrated that synchronized release of SCNderived vasopressin, and ARC-derived $\alpha-\mathrm{MSH}$ in the medial preoptic area is essential for the physiologic dawn temperature decrease in rats. According to our present observation of an altered ARC PER1 rhythm in RC-cut animals, disclosing a desynchronous state of the $A R C$ from the SCN, should indeed lead to a loss in temperature rhythm. The necessity of ARC and SCN synchrony is also consistent with data showing the ARC is essential for the regulation of activity and feeding. Lesions targeted at specific neuronal populations in the ARC resulted in deteriorated activity, temperature, and feeding rhythms (Coppari et al., 2005; Li et al., 2012). Our results indicate, it is not the ARC lesions per se, but an altered phase relationship between the SCN and ARC leading to desynchronized SCN-ARC output with a deleterious effect on physiologic function. As such, the observation that RCcut animals maintain a normal melatonin rhythm in LD and DD conditions affirms that melatonin is solely dependent on SCN activity (Pévet, 2014), while rhythms in locomotor activity, temperature, and corticosterone levels are also dependent on regulation by the $A R C$ and thus arguably affected by changes in metabolism.

\section{Technical considerations}

Making knife cuts inevitably causes collateral damage, and while keeping this to a minimum (as shown in Figures 1 and 6), we cannot exclude consequential damage to neurons and pathways to and from other areas, perchance contributing to observed rhythmic aberrations. Retrochiasmatic lesions for example, have been shown to reduce pineal melatonin content (Ribeiro Barbosa et al., 1999); however, in the present study, the rhythm in mel- atonin secretion was not altered and animals with knife cuts placed out of midline, still transecting the retrochiasmatic area, did not express any change in rhythmicity. The SPZ, located above the area of the knife cut, is a target of neural projections from the SCN (Watts et al., 1987) and functions as a possible relay for the SCN (Cipolla-Neto et al., 1988), suggesting that a loss of SPZ input could be responsible for observed change in rhythmicity. This, however, seems unlikely for two reasons; first, we show the SCN projections to the PVN, SPZ and DMH are still intact (Fig. 6). Second, other studies demonstrated that horizontal knife cuts eliminating SCN input to the SPZ reduced the LH surge in female rats but did not reduce the rhythmicity of locomotor activity, nor in constant conditions (Watts et al., 1989), indicating that the SCN projections to the SPZ are not essential for rhythm in locomotor activity.

Realizing that knife cuts could also sever unforeseen projections we investigated SCN and ARC projections to and from other hypothalamic areas. The presence of NPY input from the IGL to the SCN was unaffected and also ARC and SCN output to other areas was not notably altered. Still the possible disruption of other SCN or ARC afferents could be considered a limitation of the knife cut technique, restricting a conclusion about the SCN-ARC interaction. Thus, to reduce this possibility we performed a unilateral SCN lesion with a contralateral knife cut. Unilateral SCN lesions alone allow an animal to maintain its circadian rhythm (Gerkema et al., 1990; Guzmán-Ruiz et al., 2014). Hence, to ensure our observations were the result of severed interaction between the SCN and ARC and not due to damage of the retrochiasmatic area or other hypothalamic projections to the SCN and ARC, we combined unilateral SCN lesions with a contralateral knife cut, leaving the unilateral SCN, unilateral retrochiasmatic area and complete ARC intact. With this experiment, again preventing reciprocal communication between SCN and $\mathrm{ARC}$, we demonstrate equal aberrations of physiologic rhythms as seen following a complete $R C$ cut. In combination with demonstrated intact SCN (VIP) and ARC (AgRP) efferents to other hypothalamic target areas, we show it is the eliminated SCN-ARC communication responsible for observed loss of rhythmicity in physiologic outputs and not damage to other hypothalamic nuclei or circuits. Since we investigated the nature of SCN-ARC interaction consisting of various, unknown, neuronal populations, a Cre recombinase animal study would not be appropriate for this specific purpose. However, it could prove applicable in future research, investigating the character of involved individual neuronal populations and their function in SCN-ARC interaction.

\section{The SCN and ARC depend on interaction to modulate their activity in response to metabolic cues}

The rhythmic SCN Per1 expression together with an intact melatonin rhythm demonstrates preserved SCN rhythmicity. However, RC-cut animals responded to metabolic cues not only with an altered neuronal activation of the ARC but also of the SCN, illustrating that for proper function both the ARC and SCN depend on their intercon- 
nectivity through the SCN-ARC axis. This is consistent with observations that altered metabolic conditions of an animal change the activity of the ARC and that of the SCN simultaneously (Yi et al., 2008; Saderi et al., 2013). Moreover, recent findings show that the $S C N$ readily modulates glucose sensing in the ARC in a time dependent manner (Chao et al., 2016) and light not only directly changes the neuronal activity of the SCN but also that of the ARC (Guzmán-Ruiz et al., 2014) further confirming the significance of the SCN-ARC axis.

\section{Conclusion}

The present results provide evidence that SCN-ARC interaction serves to synchronize SCN and ARC output, essential for organizing physiologic functions. This confirms the idea, also previously suggested (Webb et al., 2009; Hu et al., 2012; Buijs et al., 2016), that the SCN functions inside a larger circadian network of tightly linked oscillatory feedback circuits whose integral function is essential for regulating physiologic and behavioral functions. Long-term desynchronization within this circadian network due to changes in dietary habits, chronic jetlag, or shift work is known to contribute to pathology associated with "modern lifestyle," such as hypertension, obesity, diabetes, and cancer (Scheer et al., 2010; Leproult et al., 2014; Kettner et al., 2016). We therefore propose that ill-timed food intake and altered metabolic signals are able to alter normal ARC activity patterns, as such changing its synchronization with the SCN and as a consequence disrupting associated behavioral and hormonal patterns. Thus, faulty network connections or erroneous feedback may reshape the circadian system to a new equilibrium, leading to physiologic impairment and pathology.

\section{References}

Abe H, Kida M, Tsuji K, Mano T (1989) Feeding cycles entrain circadian rhythms of locomotor activity in CS mice but not in C57BL/6J mice. Physiol Behav 45:397-401. CrossRef

Acosta-Galvan G, Yi CX, Van der Vilet J, Jhamandas JH, Panula P, Angeles-Castellanos M, Del Carmen BM, Escobar C, Buijs RM (2011) Interaction between hypothalamic dorsomedial nucleus and the suprachiasmatic nucleus determines intensity of food anticipatory behavior. Proc Natl Acad Sci USA 108:5813-5818. CrossRef

Antle MC, Mistlberger RE (2000) Circadian clock resetting by sleep deprivation without exercise in the Syrian hamster. J Neurosci 20:9326-9332. Medline

Armstrong SM (1989) Melatonin and circadian control in mammals. Experientia 45:932-938. Medline

Buijs FN, Cazarez F, Basualdo MC, Scheer FA, Perusquía M, Centurion D, Buijs RM (2014) The suprachiasmatic nucleus is part of a neural feedback circuit adapting blood pressure response. Neuroscience 266:197-207. CrossRef Medline

Buijs FN, León-Mercado L, Guzmán-Ruiz M, Guerrero-Vargas NN, Romo-Nava F, Buijs RM (2016) The circadian system: a regulatory feedback network of periphery and brain. Physiology (Bethesda) 31:170-181. CrossRef Medline

Buijs RM, Pool CW, Van Heerikhuize JJ, Sluiter AA, Van Der Sluis PJ, Ramkema M, Van Der Woude TP, Van Der Beek E (1989) Antibodies to small transmitter molecules and peptides: production and application of antibodies to dopamine, serotonin, GABA, vasopressin, vasoactive intestinal polypeptide, neuropeptide $\mathrm{Y}$, somatostatin and substance. Biomed Res 10:213-221.
Chao DH, Leon-Mercado L, Foppen E, Guzman-Ruiz M, Basualdo MC, Escobar C, Buijs RM (2016) The Suprachiasmatic nucleus modulates the sensitivity of Arcuate nucleus to hypoglycemia in the male rat. Endocrinologyen 157:3439-3451.

Cipolla-Neto J, Afeche SC, Menna-Barreto L, Marques N, BeneditoSilva AA, Fortunato G, Recine EG, Schott C (1988) Lack of similarity between the effect of lesions of the suprachiasmatic nucleus and subparaventricular hypothalamic zone on behavioral circadian rhythms. Braz J Med Biol Res 21:653-654. Medline

Coppari R, Ichinose M, Lee CE, Pullen AE, Kenny CD, McGovern RA, Tang V, Liu SM, Ludwig T, Chua SC Jr, Lowell BB, Elmquist JK (2005) The hypothalamic arcuate nucleus: a key site for mediating leptin's effects on glucose homeostasis and locomotor activity. Cell Metab 1:63-72. CrossRef

Edgar DM, Kilduff TS, Martin CE, Dement WC (1991) Influence of running wheel activity on free-running sleep/wake and drinking circadian rhythms in mice. Physiol Behav 50:373-378. Medline

Gerkema MP, Groos GA, Daan S (1990) Differential elimination of circadian and ultradian rhythmicity by hypothalamic lesions in the common vole, Microtus arvalis. J Biol Rhythms 5:81-95. CrossRef Medline

Guerrero-Vargas NN, Salgado-Delgado R, Basualdo MD, Garcia J, Guzman-Ruiz M, Carrero JC, Escobar C, Buijs RM (2014) Reciprocal interaction between the suprachiasmatic nucleus and the immune system tunes down the inflammatory response to lipopolysaccharide. J Neuroimmunol 273:22-30.

Guzmán-Ruiz M, Saderi N, Cazarez-Márquez F, Guerrero-Vargas NN, Basualdo MC, Acosta-Galván G, Buijs RM (2014) The suprachiasmatic nucleus changes the daily activity of the arcuate nucleus alpha-MSH neurons in male rats. Endocrinology 155:525535. CrossRef Medline

Guzman-Ruiz MA, Ramirez-Corona A, Guerrero-Vargas NN, Sabath E, Ramirez-Plascencia OD, Fuentes-Romero R, Leon-Mercado LA, Basualdo SM, Escobar C, Buijs RM (2015) Role of the suprachiasmatic and arcuate nuclei in diurnal temperature regulation in the rat. J Neurosci 35:15419-15429. CrossRef

Herrera-Moro CD, León-Mercado L, Foppen E, Guzmán-Ruiz M, Basualdo MC, Escobar C, Buijs RM (2016) The suprachiasmatic nucleus modulates the sensitivity of arcuate nucleus to hypoglycemia in the male rat. Endocrinology 157:3439-3451. CrossRef Medline

Houdek P, Nováková M, Polidarová L, Sládek M, Sumová A (2016) Melatonin is a redundant entraining signal in the rat circadian system. Horm Behav 83:1-5. CrossRef

Hu K, Meijer JH, Shea SA, Vanderleest HT, Pittman-Polletta B, Houben T, van Oosterhout F, Deboer T, Scheer FA (2012) Fractal patterns of neural activity exist within the suprachiasmatic nucleus and require extrinsic network interactions. PLoS One 7:e48927. CrossRef Medline

Janik D, Mrosovsky N (1994) Intergeniculate leaflet lesions and behaviorally-induced shifts of circadian rhythms. Brain Res 651: 174-182. Medline

Kettner NM, Voicu H, Finegold MJ, Coarfa C, Sreekumar A, Putluri N, Katchy CA, Lee C, Moore DD, Fu L (2016) Circadian homeostasis of liver metabolism suppresses hepatocarcinogenesis. Cancer Cell 30:909-924. CrossRef Medline

Kriegsfeld LJ, Lesauter J, Silver R (2004) Targeted microlesions reveal novel organization of the hamster suprachiasmatic nucleus. J Neurosci 24:2449-2457. CrossRef Medline

Lamont EW, Diaz LR, Barry-Shaw J, Stewart J, Amir S (2005) Daily restricted feeding rescues a rhythm of period2 expression in the arrhythmic suprachiasmatic nucleus. Neuroscience 132:245-248. CrossRef Medline

Leon-Mercado L, Herrera Moro CD, Basualdo MD, Kawata M, Escobar C, Buijs RM (2017) The arcuate nucleus: a site of fast negative feedback for corticosterone secretion in male rats. eNeuro 4:ENEURO-16. CrossRef

Leproult R, Holmback U, Van Cauter E (2014) Circadian misalignment augments markers of insulin resistance and inflammation, independently of sleep loss. Diabetes 63:1860-1869. 
Li AJ, Wiater MF, Oostrom MT, Smith BR, Wang Q, Dinh TT, Roberts BL, Jansen HT, Ritter S (2012) Leptin-sensitive neurons in the arcuate nuclei contribute to endogenous feeding rhythms. Am J Physiol Regul Integr Comp Physiol 302:R1313-R1326. CrossRef

Marchant EG, Mistlberger RE (1995) Morphine phase-shifts circadian rhythms in mice: role of behavioural activation. Neuroreport 7:209-212. Medline

Mendoza J (2007) Circadian clocks: setting time by food. J Neuroendocrinol 19:127-137. CrossRef Medline

Moore RY, Eichler VB (1972) Loss of a circadian adrenal corticosterone rhythm following suprachiasmatic lesions in the rat. Brain Res 42:201-206. Medline

O'Callaghan EK, Anderson ST, Moynagh PN, Coogan AN (2012) Long-lasting effects of sepsis on circadian rhythms in the mouse. PLoS One 7:e47087.

Oster H, Damerow S, Hut RA, Eichele G (2006) Transcriptional profiling in the adrenal gland reveals circadian regulation of hormone biosynthesis genes and nucleosome assembly genes. J Biol Rhythms 21:350-361. CrossRef

Peters RV, Zoeller RT, Hennessey AC, Stopa EG, Anderson G, Albers HE (1994) The control of circadian rhythms and the levels of vasoactive intestinal peptide messenger RNA in the suprachiasmatic nucleus are altered in spontaneously hypertensive rats. Brain Res 639:217-227. Medline

Pévet P (2014) The internal time-giver role of melatonin. A key for our health. Rev Neurol (Paris) 170:646-652. CrossRef

Pitrosky B, Kirsch R, Malan A, Mocaer E, Pevet P (1999) Organization of rat circadian rhythms during daily infusion of melatonin or S20098, a melatonin agonist. Am J Physiol 277:R812-R828. Medline

Quintero JE, Kuhlman SJ, Mcmahon DG (2003) The biological clock nucleus: a multiphasic oscillator network regulated by light. J Neurosci 23:8070-8076.

Redlin U, Mrosovsky N (1999) Masking of locomotor activity in hamsters. J Comp Physiol A Neuroethol Sens Neural Behav Physiol 184:429-437. Medline

Reppert SM, Weaver DR (2002) Coordination of circadian timing in mammals. Nature 418:935-941. CrossRef Medline

Ribeiro Barbosa ER, Skorupa AL, Cipolla Neto J, Canteras NS (1999) Projections of the basal retrochiasmatic area: a neural site involved in the photic control of pineal metabolism. Brain Res 839:35-40. Medline

Saderi N, Cazarez-Marquez F, Buijs FN, Salgado-Delgado RC, Guzman-Ruiz MA, Del Carmen BM, Escobar C, Buijs RM (2013) The NPY intergeniculate leaflet projections to the suprachiasmatic nucleus transmit metabolic conditions. Neuroscience 246:291300. CrossRef

Saderi N, Salgado-Delgado R, Avendano-Pradel R, Basualdo MC, Ferri GL, Chávez-Macías L, Roblera JE, Escobar C, Buijs RM (2012) NPY and VGF immunoreactivity increased in the arcuate nucleus, but decreased in the nucleus of the tractus solitarius, of type-II diabetic patients. PLoS One 7:e40070. CrossRef
Saeb-Parsy K, Lombardelli S, Khan FZ, McDowall K, Au-Yong IT, Dyball RE (2000) Neural connections of hypothalamic neuroendocrine nuclei in the rat. J Neuroendocrinol 12:635-648.

Schaap J, Meijer JH (2001) Opposing effects of behavioural activity and light on neurons of the suprachiasmatic nucleus. Eur $\mathrm{J}$ Neurosci 13:1955-1962. CrossRef

Scheer FA, Hu K, Evoniuk H, Kelly EE, Malhotra A, Hilton MF, Shea SA (2010) Impact of the human circadian system, exercise, and their interaction on cardiovascular function. Proc Natl Acad Sci USA 107:20541-20546. CrossRef Medline

Shieh KR, Yang SC, Lu XY, Akil H, Watson SJ (2005) Diurnal rhythmic expression of the rhythm-related genes, rPeriod1, rPeriod2, and rClock, in the rat brain. J Biomed Sci 12:209-217. CrossRef

Shioiri T, Takahashi K, Yamada N, Takahashi S (1991) Motor activity correlates negatively with free-running period, while positively with serotonin contents in SCN in free-running rats. Physiol Behav 49:779-786. Medline

Silver R, Lesauter J, Tresco PA, Lehman MN (1996) A diffusible coupling signal from the transplanted suprachiasmatic nucleus controlling circadian locomotor rhythms. Nature 382:810-813. CrossRef Medline

Vansteensel MJ, Yamazaki S, Albus H, Deboer T, Block GD, Meijer $\mathrm{JH}$ (2003) Dissociation between circadian Per1 and neuronal and behavioral rhythms following a shifted environmental cycle. Curr Biol 13:1538-1542. Medline

Watts AG, Sheward WJ, Whale D, Fink G (1989) The effects of knife cuts in the sub-paraventricular zone of the female rat hypothalamus on oestrogen-induced diurnal surges of plasma prolactin and LH, and circadian wheel-running activity. J Endocr 122:593-604. Medline

Watts AG, Swanson LW, Sanchez-Watts G (1987) Efferent projections of the Suprachiasmatic nucleus: I. Studies using anterograde transport of Phaseolus vulgaris leucoagglutinin in the rat. J Comp Neur 258:204-229. CrossRef

Webb AB, Angelo N, Huettner JE, Herzog ED (2009) Intrinsic, nondeterministic circadian rhythm generation in identified mammalian neurons. Proc Natl Acad Sci USA 106:16493-16498. CrossRef Medline

Wiater MF, Li AJ, Dinh TT, Jansen HT, Ritter S (2013) Leptin-sensitive neurons in the arcuate nucleus integrate activity and temperature circadian rhythms and anticipatory responses to food restriction. Am J Physiol Regul Integr Comp Physiol 305:R949-R960. CrossRef

Yamazaki S, Kerbeshian MC, Hocker CG, Block GD, Menaker M (1998) Rhythmic properties of the hamster suprachiasmatic nucleus in vivo. J Neurosci 18:10709-10723. Medline

Yi CX, Challet E, Pévet P, Kalsbeek A, Escobar C, Buijs RM (2008) A circulating ghrelin mimetic attenuates light-induced phase delay of mice and light-induced Fos expression in the suprachiasmatic nucleus of rats. Eur J Neurosci 27:1965-1972. CrossRef Medline

Yi CX, Van D, V, Dai J, Yin G, Ru L, Buijs RM (2006) Ventromedial arcuate nucleus communicates peripheral metabolic information to the suprachiasmatic nucleus. Endocrinology 147:283-294. CrossRef 